CERN-TH.7300/94

hep-ph/9406274

\title{
One-Particle Inclusive Processes in Deeply Inelastic Lepton-Nucleon Scattering
}

\author{
Dirk Graudenz \\ Theoretical Physics Division, CERN \\ CH-1211 Geneva 23
}

\begin{abstract}
The one-particle inclusive cross section in deeply inelastic lepton-nucleon scattering, expressed in terms of parton densities and fragmentation functions being differential in the invariant mass of the observed hadron and of the incoming nucleon, diverges if this invariant mass vanishes. This divergence can be traced back to the kinematical configuration where the parent parton of the observed hadron is emitted collinearly from the incoming parton of the QCD subprocess. By using the concept of "fracture functions", which has recently been introduced by Trentadue and Veneziano, it is possible to absorb this divergence in these new distribution functions as long as the observed hadron is not soft. This procedure allows the determination of a finite one-particle inclusive cross section in next-to-leading order QCD perturbation theory. We give details of the calculation and the explicit form of the bare fracture functions in terms of the renormalized ones.
\end{abstract}

CERN-TH.7300/94

June 1994

\footnotetext{
${ }^{\ddagger}$ Electronic mail address: graudenz@cernvm.cern.ch
} 


\section{Introduction}

QCD as a theory of the strong interactions should be able to give a description of one-particle inclusive processes. Since non-perturbative effects such as the nucleon wave functions and the fragmentation of quarks and gluons are not manageable in explicit calculations, it is necessary to parametrize them by means of phenomenological distribution functions in order to make contact with perturbative QCD [1, 2, 3, 4, 5, 6, 7]. The case of one-particle inclusive lepton-nucleon scattering has been considered by several authors [8, 9, 5, 6, 10, 11, 12, 13]. The canonical procedure to do the calculation is to define a variable $z_{\mathrm{h}}=P_{0} h / P_{0} q$ as in [5], where $P_{0}$ is the momentum of the incoming nucleon, $q$ is the momentum of the exchanged virtual photon, and $h$ is the momentum of the observed hadron. It turns out that $\mathrm{d} \sigma / \mathrm{d} z_{\mathrm{h}}$, the differential cross section for the production of $\mathrm{h}$ plus anything else, is singular for $z_{\mathrm{h}} \rightarrow 0$. A similar effect $\left(\mathrm{d} \sigma / \mathrm{d} z_{\mathrm{h}} \rightarrow \infty\right.$ for $\left.z_{\mathrm{h}} \rightarrow 0\right)$ can be observed in the case of $\mathrm{e}^{+} \mathrm{e}^{-}$annihilation, where $z_{\mathrm{h}} \rightarrow 0$ means that the observed hadron $\mathrm{h}$ is soft $\left(E_{\mathrm{h}} \rightarrow 0\right)$. This singularity is physical, being related to the fact that the multiplicity for the emission of soft particles is infinite. However, in lepton-nucleon scattering the situation is different: $z_{\mathrm{h}} \rightarrow 0$ means (in the case of massless particles) that either $E_{\mathrm{h}} \rightarrow 0$ or that $\mathrm{h}$ is collinear with the target remnant. In the case $E_{\mathrm{h}} \rightarrow 0$ the same argument as in the $\mathrm{e}^{+} \mathrm{e}^{-}$case applies. If, however, $z_{\mathrm{h}} \rightarrow 0$, but not $E_{\mathrm{h}} \rightarrow 0$, the process under consideration is that of a hadron $h$ with non-vanishing energy emitted in the remnant direction, which is physically perfectly well defined and has to be distinguished from the case of a soft process. It is possible to separate the two cases by introducing energy and angle variables $z$ and $v(z=0$ means $E_{\mathrm{h}}=0, v=1$ means that $\mathrm{h}$ is collinear with the remnant) for the observed hadron. This allows to consider the case of $z_{\mathrm{h}} \rightarrow 0$ without having $E_{\mathrm{h}} \rightarrow 0 . \mathrm{d} \sigma / \mathrm{d} z \mathrm{~d} v$ can be calculated in perturbative QCD by means of parton densities and fragmentation functions. Again, it turns out that $\mathrm{d} \sigma / \mathrm{d} z \mathrm{~d} v \rightarrow \infty$ for $z \neq 0, v \rightarrow 1$. A closer analysis of the problem reveals that it stems from the contributions in which the fragmentation function is attached to an outgoing parton which is emitted from the incoming parton. It is clear that a collinear divergence appears if the mentioned outgoing parton is collinear to the incoming one. In this phase-space region, the observed hadron $\mathrm{h}$ comes from the target remnant region. This singularity cannot be absorbed into the parton distribution functions or the fragmentation functions since in lowest order (in the formalism based solely on parton densities and fragmentation functions) the observed hadron always originates in the current region. To absorb the divergence, it is necessary to introduce a distribution function that describes hadrons in the target remnant region. This has been done in [14]; the new distribution functions have been called "fracture functions". In this paper it is shown that, using these concepts, a finite cross section $\mathrm{d} \sigma / \mathrm{d} z \mathrm{~d} v$ for deeply inelastic lepton-nucleon scattering can be calculated for $E_{\mathrm{h}} \neq 0$ in next-to-leading order QCD perturbation theory.

The outline of this paper is as follows. In Section 2 the calculation of one-particle inclusive cross sections is briefly reviewed. The $\mathcal{O}\left(\alpha_{s}\right)$ QCD corrections to the parton-model process are determined in Section 3. In Section 4 the explicit form of the renormalized fracture function in terms of bare distribution functions is stated. The finite cross section is discussed in Section 5 . The paper closes with a summary and some conclusions. Technical details and explicit formulas are relegated to appendices. 


\section{One-Particle Inclusive Cross Sections}

In the following the scattering process

$$
\mathrm{l}+\mathrm{A} \rightarrow \mathrm{l}^{\prime}+\mathrm{h}+\mathrm{X}
$$

is considered, where $\mathrm{l}$ and $\mathrm{A}$ are the incoming charged lepton and nucleon, respectively, $\mathrm{l}^{\prime}$ is the scattered charged lepton, $\mathrm{h}$ is the identified hadron, and $\mathrm{X}$ denotes anything else in the hadronic final state. Let $l$ and $l^{\prime}$ be the momenta of the incoming and outgoing lepton, respectively, $P_{0}$ the momentum of the incoming nucleon and $h$ the momentum of the outgoing identified hadron. The integration over the angles that determine the relative orientation of the leptonic and hadronic final state can be performed. The remaining lepton phase-space variables are the Bjorken variables

$$
x_{B}=\frac{Q^{2}}{2 P_{0} q}, \quad y=\frac{P_{0} q}{P_{0} l} .
$$

Here $q=l-l^{\prime}$ is the momentum transfer and $Q^{2}=-q^{2}>0$ is the virtuality of the exchanged virtual photon.

The cross section for the production of an $n$-parton final state differential in $x_{B}, y$ and the phase-space of the final-state partons is

$$
\frac{\mathrm{d} \sigma}{\mathrm{d} x_{B} \mathrm{~d} y \mathrm{dPS}(n)}=\sum_{i} \int \frac{\mathrm{d} \xi}{\xi} P_{i / \mathrm{A}}(\xi) \alpha^{2} \frac{1}{2 S_{H} x_{B}} \frac{1}{e^{2}(2 \pi)^{2 d}}\left(Y^{M}\left(-g^{\mu \nu}\right)+Y^{L} \frac{4 x_{B}{ }^{2}}{Q^{2}} P_{0}^{\mu} P_{0}^{\nu}\right) H_{\mu \nu} .
$$

Here the case of one-photon exchange has been considered; the exchange of weak vector bosons is neglected for simplicity. $S_{H}=\left(P_{0}+l\right)^{2}$ is the square of the total CM energy of the lepton-nucleon scattering process, $\xi$ is the momentum fraction of the initial parton of the QCD subprocess, and $P_{i / \mathrm{A}}(\xi)$ is the probability distribution function for parton $i$ in the nucleon $\mathrm{A} ; P_{i / \mathrm{A}}(\xi)$ can be either a parton density $f_{i / \mathrm{A}}(\xi)$ or a fracture function $M_{i, h / \mathrm{A}}(\xi, \zeta)$, see below. $\alpha=e^{2} / 4 \pi$ is the fine structure constant and $d=4-2 \epsilon$ is the space-time dimension used in dimensional regularization. A factor of $1 / 4$ for the average over the spin degrees of freedom of the incoming particles is already included. The last factor, $H_{\mu \nu}$, is the hadron tensor defined by

$$
H_{\mu \nu}=\sum_{\text {spins }} \overline{\mathcal{M}_{\mu}} \mathcal{M}_{\nu}
$$

where $\epsilon^{\mu}(\lambda) \mathcal{M}_{\mu}$ is the matrix element for the process

$$
\gamma^{*}+\text { parton } \rightarrow n \text { partons }
$$

with $\epsilon^{\mu}(\lambda)$ the polarization vector of a virtual photon with polarization $\lambda$. The ratios

$$
Y^{M}=\frac{1+(1-y)^{2}}{2 y^{2}} \quad \text { and } \quad Y^{L}=\frac{4(1-y)+1+(1-y)^{2}}{2 y^{2}}
$$

specify the $y$-dependence of the contributions from the two photon polarizations. The projections operating on $H_{\mu \nu}$ are the result of the integration over the angles that describe the lepton-hadron orientation. The cross section consists of two parts, proportional to $Y^{M}$, the "metric" contribution 
(it is obtained by a contraction of the hadron tensor with $\left(-g^{\mu \nu}\right)$ ), and proportional to $Y^{L}$, the longitudinal contribution.

For the production of an identified hadron $\mathrm{h}$ in the final state, there are in principle two mechanisms [15]: $\mathrm{h}$ may originate from the current (in the parton-model, from the produced quark, or in the QCD-improved parton-model, from one of the produced partons of the QCD subprocess) or from the target remnant [14], so that

$$
\sigma=\sigma_{\text {current }}+\sigma_{\text {target }} .
$$

These two contributions cannot be disentangled in the QCD-improved parton-model, because $\sigma_{\text {current }}$ gives a contribution to $\sigma_{\text {target }}$ in the case of collinear singularities in the initial state. This fact is reflected in the definition of the renormalized fracture functions, which are, contrary to the case of parton densities, inhomogeneous as a function of the bare fracture functions, as can be seen in Section 4 . In the QCD-improved parton-model, $\sigma_{\text {current }}$ and $\sigma_{\text {target }}$ are given by the graphs in Figs. 1 (a) and 1 (b), respectively; $\sigma_{\text {current }}$ can be described by means of parton densities $f_{i / \mathrm{A}}(\xi)$ and fragmentation functions $D_{h / i}(z)$. The lowest-order contribution is given by the graph in Fig. 2 (a). $\sigma_{\text {target }}$, however, needs a new phenomenological input, the "fracture function" $M_{i, h / \mathrm{A}}(\xi, \zeta)$, giving the probability to find a parton $i$ with momentum fraction $\xi$ and a hadron $\mathrm{h}$ with momentum fraction $\zeta$ in the nucleon A, for the parton model process see Fig. 2 (b). The kinematical restriction on $\xi$ and $\zeta$ is $\xi+\zeta \leq 1$.

It is reasonable to consider the production process in the CM frame of the incoming nucleon and the incoming virtual photon, so $\vec{P}_{0}+\vec{q}=\overrightarrow{0}$. The positive $z$-axis is defined by the $q$-direction. The hadron h has polar angle $\vartheta$ relative to the virtual photon and energy $E_{\mathrm{h}}$ in this frame. The energy of the incoming nucleon is

$$
E_{0}=\frac{Q}{2} \frac{1}{\sqrt{x_{B}\left(1-x_{B}\right)}}
$$

Two new variables $v, z$ can be defined by 1

$$
v=\frac{1}{2}(1-\cos \vartheta), \quad z=\frac{E_{\mathrm{h}}}{E_{0}\left(1-x_{B}\right)}
$$

The range of these variables is $v, z \in[0,1]$ if the masses of all particles are neglected. The differential cross section calculated in the following is

$$
\frac{\mathrm{d} \sigma\left(\mathrm{l}+\mathrm{A} \rightarrow \mathrm{l}^{\prime}+\mathrm{h}+\mathrm{X}\right)}{\mathrm{d} x_{B} \mathrm{~d} y \mathrm{~d} z \mathrm{~d} v}
$$

It turns out that QCD-corrections to the lowest-order process require subtractions in the collinear region that make this differential cross section a distribution instead of a function of the variable $v$. It is therefore more reasonable to consider an observable $A(v)$ and to integrate over $v$

$$
\langle A\rangle=\int_{0}^{1} \mathrm{~d} v \frac{\mathrm{d} \sigma}{\mathrm{d} v} A(v) .
$$

\footnotetext{
${ }^{1}$ The variable $z$ has no relation to the variable $z_{\mathrm{h}}$ mentioned in the introduction.
} 
This way one defines the expectation value

$$
\mathcal{A}=\frac{\mathrm{d}\langle A\rangle}{\mathrm{d} x_{B} \mathrm{~d} y \mathrm{~d} z}
$$

Explicitly, it is given by

$$
\begin{aligned}
\mathcal{A}= & \sum_{j} \int \frac{\mathrm{d} u}{u} \sum_{N} \sum_{\underline{i}} \int \mathrm{dPS}^{(N)}(\underline{p}) \frac{\alpha^{2}}{2 S_{H} x_{B}} \frac{1}{e^{2}(2 \pi)^{d}} \\
\cdot & {\left[Y^{M}\left(-g^{\mu \nu}\right)+Y^{L} \frac{4 x_{B}{ }^{2}}{Q^{2}} P_{0}^{\mu} P_{0}^{\nu}\right] H_{\mu \nu} } \\
& \cdot\left\{M_{j, h / \mathrm{A}}\left(\frac{x_{B}}{u}, \frac{E_{\mathrm{h}}}{E_{0}}\right)\left(1-x_{B}\right) A(1)+f_{j / \mathrm{A}}\left(\frac{x_{B}}{u}\right) \sum_{\alpha=1}^{N} D_{\mathrm{h} / i_{\alpha}}\left(\frac{E_{\mathrm{h}}}{E_{\alpha}}\right) \frac{E_{0}}{E_{\alpha}}\left(1-x_{B}\right) A\left(v_{\alpha}\right)\right\} .
\end{aligned}
$$

The notation in this formula is the following: $N$ is the number of final-state partons in the QCD subprocess, $u=x_{B} / \xi, \underline{i}$ is a multi-index specifying the parton configuration, so $\underline{i}=\left(i_{1}, i_{2}, \ldots, i_{N}\right)$, where $i_{\alpha} \in\{u, \bar{u}, d, \bar{d}, \ldots, g\}, \underline{p}=\left(p_{1}, p_{2}, \ldots, p_{N}\right)$ is the set of momenta of the outgoing partons, $E_{\alpha}$ is the energy of the $\alpha^{\text {th }}$ parton in the $\left(\vec{P}_{0}+\vec{q}=\overrightarrow{0}\right)$ frame, and $v_{\alpha}=\left(1-\cos \vartheta_{\alpha}\right) / 2$, where $\vartheta_{\alpha}$ is the polar angle of the $\alpha^{\text {th }}$ parton in the same frame; $M, f$ and $D$ are the fracture functions, the parton densities and the fragmentation functions, respectively.

It is convenient to define the following constants and projection operators:

$$
\begin{aligned}
c_{i} & =\frac{\alpha^{2}}{2 S_{H} x_{B}} \cdot 2 \pi \cdot 4(1-\epsilon) Q_{i}^{2}, \\
P_{M}^{\mu \nu} & =\left(-g^{\mu \nu}\right), \quad P_{L}^{\mu \nu}=\frac{4 x_{B}{ }^{2}}{Q^{2}} P_{0}^{\mu} P_{0}^{\nu} .
\end{aligned}
$$

Here, $e Q_{i}$ is the electric charge of the quark of flavour $i$.

The leading order given by the processes of the parton-model depicted in Figs. 2 (a) and 2 (b) is

$$
\begin{aligned}
\mathcal{A}_{\mathrm{LO}}= & Y^{M} \sum_{i=q, \bar{q}} c_{i} \\
\cdot & \left\{\int_{\frac{x_{B}}{1-\left(1-x_{B}\right) z}}^{1} \frac{\mathrm{d} u}{u} M_{i, \mathrm{~h} / \mathrm{A}}\left(\frac{x_{B}}{u},\left(1-x_{B}\right) z\right) \delta(1-u)\left(1-x_{B}\right) A(1)\right. \\
& \left.+\int_{x_{B}}^{1} \frac{\mathrm{d} u}{u} \int \frac{\mathrm{d} \rho}{\rho} f_{i / \mathrm{A}}\left(\frac{x_{B}}{u}\right) D_{\mathrm{h} / i}\left(\frac{z}{\rho}\right) \delta(1-u) \delta(1-\rho) A(0)\right\}
\end{aligned}
$$

To this order, there are no longitudinal contributions. They arise in the QCD-improved parton-model in $\mathcal{O}\left(\alpha_{s}\right)$, which is considered in the next section.

\section{The Cross Section to $\mathcal{O}\left(\alpha_{s}\right)$}

This section gives the details of the calculation of the $\mathcal{O}\left(\alpha_{s}\right)$ corrections to the parton-model processes. The virtual 1-loop corrections to the leading-order QCD subprocess are shown in Fig. 3, and the real corrections in Figs. 4 (a),(b). 
The overall effect of the virtual corrections is to multiply the leading-order cross section by 16]

$$
1+\frac{\alpha_{s}}{2 \pi}\left(\frac{4 \pi \mu^{2}}{Q^{2}}\right)^{\epsilon} \frac{\Gamma(1-\epsilon)}{\Gamma(1-2 \epsilon)} C_{F}\left(-2 \frac{1}{\epsilon^{2}}-3 \frac{1}{\epsilon}-8-\frac{\pi^{2}}{3}\right),
$$

so

$$
\begin{aligned}
\mathcal{A}_{\mathrm{LO}+\text { virt. }}= & Y^{M} \sum_{i=q, \bar{q}} c_{i} \\
\cdot & \left\{\int_{\frac{x_{B}}{1-\left(1-x_{B}\right) z}}^{1} \frac{\mathrm{d} u}{u} M_{i, \mathrm{~h} / \mathrm{A}}\left(\frac{x_{B}}{u},\left(1-x_{B}\right) z\right) \delta(1-u)\left(1-x_{B}\right) A(1)\right. \\
& \left.+\int_{x_{B}}^{1} \frac{\mathrm{d} u}{u} \int \frac{\mathrm{d} \rho}{\rho} f_{i / \mathrm{A}}\left(\frac{x_{B}}{u}\right) D_{\mathrm{h} / i}\left(\frac{z}{\rho}\right) \delta(1-u) \delta(1-\rho) A(0)\right\} \\
& \left\{11+\frac{\alpha_{s}}{2 \pi}\left(\frac{4 \pi \mu^{2}}{Q^{2}}\right)^{\epsilon} \frac{\Gamma(1-\epsilon)}{\Gamma(1-2 \epsilon)} C_{F}\left(-2 \frac{1}{\epsilon^{2}}-3 \frac{1}{\epsilon}-8-\frac{\pi^{2}}{3}\right)\right\},
\end{aligned}
$$

where $\mu$ is the renormalization scale, $\alpha_{s}=\alpha_{s}\left(\mu^{2}\right)$, and $C_{F}=4 / 3$ is one of the Casimir invariants of the colour gauge group $\mathrm{SU}(3)$.

The double and single poles in $\epsilon$ represent an infrared divergence, which is cancelled by a contribution similar to the real corrections but of opposite sign.

The projections of the hadron tensor for the real corrections are (see, for example, [17])

$$
\begin{aligned}
& \frac{1}{e^{2}(2 \pi)^{2 d}} P_{M}^{\mu \nu} H_{\mu \nu}\left(\gamma^{*} q \rightarrow q g\right) \\
& =8 \pi \frac{\alpha_{s}}{2 \pi} \mu^{2 \epsilon} 2 \pi C_{F} Q_{q}^{2} 4(1-\epsilon)\left[(1-\epsilon)\left(\frac{s_{i g}}{s_{q g}}+\frac{s_{q g}}{s_{i g}}\right)+\frac{2 Q^{2} s_{i q}}{s_{i g} s_{q g}}+2 \epsilon\right] \\
& \frac{1}{e^{2}(2 \pi)^{2 d}} P_{L}^{\mu \nu} H_{\mu \nu}\left(\gamma^{*} q \rightarrow q g\right) \\
& =8 \pi \frac{\alpha_{s}}{2 \pi} \mu^{2 \epsilon} 2 \pi C_{F} Q_{q}^{2} 4(1-\epsilon)\left[4 \frac{u^{2}}{Q^{2}} \frac{1}{2} s_{i q}\right] \\
& \frac{1}{e^{2}(2 \pi)^{2 d}} P_{M}^{\mu \nu} H_{\mu \nu}\left(\gamma^{*} g \rightarrow q \bar{q}\right) \\
& =8 \pi \frac{\alpha_{s}}{2 \pi} \mu^{2 \epsilon} 2 \pi \frac{1}{2} Q_{q}^{2} 4(1-\epsilon)\left[\frac{s_{i q}}{s_{i \bar{q}}}+\frac{s_{i \bar{q}}}{s_{i q}}-\frac{1}{1-\epsilon} \frac{2 Q^{2} s_{q \bar{q}}}{s_{i q} s_{i \bar{q}}}-2 \frac{\epsilon}{1-\epsilon}\right] \\
& \frac{1}{e^{2}(2 \pi)^{2 d}} P_{L}^{\mu \nu} H_{\mu \nu}\left(\gamma^{*} g \rightarrow q \bar{q}\right) \\
& =8 \pi \frac{\alpha_{s}}{2 \pi} \mu^{2 \epsilon} 2 \pi \frac{1}{2} Q_{q}^{2} 4(1-\epsilon)\left[4 \frac{u^{2}}{Q^{2}} s_{q \bar{q}}\right] .
\end{aligned}
$$

The invariants are $s_{A B}=2 p_{A} p_{B}$, and $p_{i}, p_{q}, p_{\bar{q}}, p_{g}$ are the momenta of the incoming parton (quark or gluon), an outgoing quark, an outgoing antiquark and an outgoing gluon, respectively. The formulas already contain the appropriate factors for the average over the colour degrees of freedom of the incoming partons. An additional factor of $1 /(1-\epsilon)$ has been provided for the terms with an incoming gluon, because gluons have $2(1-\epsilon)$ helicity states in $4-2 \epsilon$ space-time dimensions compared to only two in the case of quarks. In order to perform the phase-space integrations, suitable parametrizations

$$
\mathrm{dPS}^{(2)}=(2 \pi)^{d} \prod_{i=1}^{2}\left(\frac{\mathrm{d} p_{i} \delta\left(p_{i}^{2}\right)}{(2 \pi)^{d-1}}\right)
$$


of the two-particle phase-space are needed. The integration variable has been chosen such that it is the one that is actually used in the factorization of the collinear singularities. The integration over irrelevant angles can be performed. The results for three phase-space parametrizations A, B and C in terms of the variables $\rho, w, u^{\prime}$ are written out in Appendix $\mathrm{A}$.

By means of these phase-space parametrizations, the integrations can be performed and the infrared and collinear singularities can be factorized. The details of this straightforward but cumbersome calculation are not given here. The results are presented in a form that explicitly shows the cancellation of the collinear singularities by means of a renormalization of the phenomenological distribution functions. The contributions are denoted by $B_{1}^{M}, B_{2}^{M}, C^{M}, B_{1}^{L}, B_{2}^{L}, C^{L}$. Here the indices in $B_{\alpha}^{A}$ and $C^{A}$ stand for

- $\alpha$ : phase-space region for the integration variable $u$,

$$
\begin{aligned}
& \alpha=1: u \in\left[x_{B}, \frac{x_{B}}{x_{B}+\left(1-x_{B}\right) z}\right] \\
& \alpha=2: u \in\left[\frac{x_{B}}{x_{B}+\left(1-x_{B}\right) z}, 1\right]
\end{aligned}
$$

- A: polarization of the virtual photon,

$A=M:$ metric contribution,

$A=L$ : longitudinal contribution.

The observed hadron in the terms $B_{\alpha}^{A}$ originates from one of the outgoing partons via a fragmentation function, the one in the terms $C^{A}$ comes from the incoming hadron via a fracture function. The $B_{\alpha}^{A}$ and $C^{A}$ terms receive contributions from the graphs from Figs. 4 (a) and (b), respectively. Throughout the calculation the $\overline{\mathrm{MS}}$ factorization scheme both for the parton densities and fracture functions and for the fragmentation functions is used. The choice of the factorization scheme defines the finite parts unambiguously. One has to choose two factorization scales, one $\left(M_{f}^{2}\right)$ for the renormalized distribution functions $f$ and $M$ for partons in the incoming nucleon, and one $\left(M_{D}^{2}\right)$ for the fragmentation functions $D$.

The explicit results for the cross sections are collected in Appendix B. One sees immediately that the IR singularities proportional to $2 / \epsilon^{2}+3 / \epsilon$ cancel among virtual and real corrections. The cancellation of the remaining collinear singularities is discussed in the next section, and the finite terms $\Phi_{X}^{A}$ from appendix B are discussed in Section 5 .

\section{Renormalized Distribution Functions}

The renormalization of the bare parton densities $f_{i / \mathrm{A}}$ and bare fragmentation functions $D_{h / i}$ is done in the $\overline{\mathrm{MS}}$ factorization scheme in the standard way [16, ]], as required by the total cross section in lepton-nucleon scattering and the one-particle inclusive cross section in $e^{+} e^{-}$scattering. The expressions for the bare densities in terms of the renormalized ones for the factorization scales $M_{f}^{2}$ and $M_{D}^{2}$ are

$$
\begin{aligned}
f_{i / \mathrm{A}}(\xi) & =\int_{\xi}^{1} \frac{\mathrm{d} u}{u}\left[\delta_{i j} \delta(1-u)+\frac{1}{\epsilon} \frac{\alpha_{s}}{2 \pi} \frac{\Gamma(1-\epsilon)}{\Gamma(1-2 \epsilon)}\left(\frac{4 \pi \mu^{2}}{M_{f}^{2}}\right)^{\epsilon} P_{i \leftarrow j}(u)\right] f_{j / \mathrm{A}}^{r}\left(\frac{\xi}{u}, M_{f}^{2}\right) \\
D_{\mathrm{h} / i}(\xi) & =\int_{\xi}^{1} \frac{\mathrm{d} u}{u}\left[\delta_{i j} \delta(1-u)+\frac{1}{\epsilon} \frac{\alpha_{s}}{2 \pi} \frac{\Gamma(1-\epsilon)}{\Gamma(1-2 \epsilon)}\left(\frac{4 \pi \mu^{2}}{M_{D}^{2}}\right)^{\epsilon} P_{j \leftarrow i}(u)\right] D_{\mathrm{h} / j}^{r}\left(\frac{\xi}{u}, M_{D}^{2}\right) .
\end{aligned}
$$

The expression for $M_{i, \mathrm{~h} / \mathrm{A}}(\xi, \zeta)$ in terms of the renormalized quantity $M_{i, \mathrm{~h} / \mathrm{A}}^{r}\left(\xi, \zeta, M_{f}^{2}\right)$ can be obtained by the requirement that all collinear divergences that are not already absorbed into $f^{r}$ and $D^{r}$ are 
absorbed into $M^{r}$. By an appropriate definition of the finite parts, the bare fracture function in terms of the renormalized fracture function in the $\overline{\mathrm{MS}}$ factorization scheme is

$$
\begin{gathered}
M_{i, \mathrm{~h} / \mathrm{A}}(\xi, \zeta)=\int_{\frac{\xi}{1-\zeta}}^{1} \frac{\mathrm{d} u}{u}\left[\delta_{i j} \delta(1-u)+\frac{1}{\epsilon} \frac{\alpha_{s}}{2 \pi} \frac{\Gamma(1-\epsilon)}{\Gamma(1-2 \epsilon)}\left(\frac{4 \pi \mu^{2}}{M_{f}^{2}}\right)^{\epsilon} P_{i \leftarrow j}(u)\right] M_{j, \mathrm{~h} / \mathrm{A}}^{r}\left(\frac{\xi}{u}, \zeta, M_{f}^{2}\right) \\
+\int_{\xi}^{\frac{\xi}{\xi+\zeta}} \frac{\mathrm{d} u}{u} \frac{1}{1-u} \frac{u}{x_{B}} \frac{1}{\epsilon} \frac{\alpha_{s}}{2 \pi} \frac{\Gamma(1-\epsilon)}{\Gamma(1-2 \epsilon)}\left(\frac{4 \pi \mu^{2}}{M_{f}^{2}}\right)^{\epsilon} \hat{P}_{k i \leftarrow j}(u) f_{j / \mathrm{A}}\left(\frac{\xi}{u}\right) D_{\mathrm{h} / k}\left(\frac{\zeta u}{\xi(1-u)}\right) .
\end{gathered}
$$

We have implicitly assumed that repeated indices are summed over. These expressions are valid to $\mathcal{O}\left(\alpha_{s}\right)$. The formula for $M$ is inhomogeneous. The homogeneous contribution is just the standard Altarelli-Parisi evolution caused by the emission of collinear partons from the parton emanating from a fracture function. The inhomogeneous term arises because the observed hadron may originate from a fragmentation function of an outgoing parton collinear to the incoming one.

By requiring that the bare fracture functions do not depend on the factorization scale and consequently

$$
\frac{\mathrm{d}}{\mathrm{d} \ln M_{f}^{2}} M_{i, \mathrm{~h} / \mathrm{A}}(\xi, \zeta)=0,
$$

the evolution equation for $M_{i, \mathrm{~h} / \mathrm{A}}^{r}\left(\xi, \zeta, M_{f}^{2}\right)$ can be derived. It coincides with that in 14.

Rewriting the bare distribution functions in terms of the renormalized ones in all cross-section formulas, and keeping only terms up to $\mathcal{O}\left(\alpha_{s}\right)$, one can see that all collinear singularities cancel. The result is a finite cross section to $\mathcal{O}\left(\alpha_{s}\right)$, which is discussed in the next section.

\section{$5 \quad$ Finite Contributions}

After the cancellation of the infrared and collinear singularities the resulting cross section is finite. Because of the subtractions, it is of the form of a convolution of a distribution with phenomenological distribution functions. The "+" prescriptions used are defined by [18]:

$$
\int \mathrm{d} x D_{+x[\underline{a}, b]}(x) \varphi(x)=\int_{a}^{b} \mathrm{~d} x D(x)(\varphi(x)-\varphi(a)) .
$$

The variable and the range of the integration are indicated as a subscript; furthermore, the subtraction

point is underlined. These distributions are used in the expressions of the $\Phi_{X}^{L / M}$, which are presented in Appendix G. The expectation value of the observable $A$ is, differential in $x_{B}, y$ and $z$ :

$$
\frac{\mathrm{d}\langle A\rangle}{\mathrm{d} x_{B} \mathrm{~d} y \mathrm{~d} z}=\mathcal{A}_{\mathrm{LO}+\text { virt. }}^{f}+\mathcal{A}_{B_{1}^{M}}^{f}+\mathcal{A}_{B_{2}^{M}}^{f}+\mathcal{A}_{C^{M}}^{f}+\mathcal{A}_{B_{1}^{L}}^{f}+\mathcal{A}_{B_{2}^{L}}^{f}+\mathcal{A}_{C^{L}}^{f}
$$

with

$$
\begin{aligned}
\mathcal{A}_{\mathrm{LO}+\text { virt. }}^{f}= & Y^{M} \sum_{i=q, \bar{q}} c_{i} \\
& \left\{\int_{\frac{x_{B}}{1-\left(1-x_{B}\right) z}}^{1} \frac{\mathrm{d} u}{u} M_{i, \mathrm{~h} / \mathrm{A}}^{r}\left(\frac{x_{B}}{u},\left(1-x_{B}\right) z, M_{f}^{2}\right) \delta(1-u)\left(1-x_{B}\right) A(1)\right. \\
& \left.+\int_{x_{B}}^{1} \frac{\mathrm{d} u}{u} \int \frac{\mathrm{d} \rho}{\rho} f_{i / \mathrm{A}}^{r}\left(\frac{x_{B}}{u}, M_{f}^{2}\right) D_{\mathrm{h} / \mathrm{A}}^{r}\left(\frac{z}{\rho}, M_{D}^{2}\right) \delta(1-u) \delta(1-\rho) A(0)\right\} \\
& \cdot\left\{1+\frac{\alpha_{s}}{2 \pi}\left(-8-\frac{\pi^{2}}{3}\right)\right\},
\end{aligned}
$$




$$
\begin{aligned}
\mathcal{A}_{B_{1}^{M}}^{f}= & Y^{M} \sum_{i=q, \bar{q}} c_{i} \frac{\alpha_{s}}{2 \pi} \int_{x_{B}}^{\frac{x_{B}}{x_{B}+\left(1-x_{B}\right) z}} \frac{\mathrm{d} u}{u} \int_{a(u)}^{1} \frac{\mathrm{d} \rho}{\rho} A(v(\rho, u)) \\
& \cdot\left[f_{i / \mathrm{A}}^{r}\left(\frac{x_{B}}{u}, M_{f}^{2}\right) D_{\mathrm{h} / i}^{r}\left(\frac{z}{\rho}, M_{D}^{2}\right)\left\{-\ln \frac{M_{f}^{2}}{Q^{2}} P_{q \leftarrow q}(u) \delta(1-\rho)+C_{F} \Phi_{1 q q}^{M}\right\}\right. \\
& +f_{i / \mathrm{A}}^{r}\left(\frac{x_{B}}{u}, M_{f}^{2}\right) D_{\mathrm{h} / g}^{r}\left(\frac{z}{\rho}, M_{D}^{2}\right)\left\{-\ln \frac{M_{f}^{2}}{Q^{2}} \hat{P}_{g q \leftarrow q}(u) \delta(\rho-a(u))+C_{F} \Phi_{1 q g}^{M}\right\} \\
& +f_{g / \mathrm{A}}^{r}\left(\frac{x_{B}}{u}, M_{f}^{2}\right) D_{\mathrm{h} / i}^{r}\left(\frac{z}{\rho}, M_{D}^{2}\right) \\
& \left.\cdot\left\{-\ln \frac{M_{f}^{2}}{Q^{2}} \hat{P}_{\bar{q} q \leftarrow g}(u) \delta(\rho-a(u))-\ln \frac{M_{f}^{2}}{Q^{2}} P_{q \leftarrow g}(u) \delta(1-\rho)+\frac{1}{2} \Phi_{1 g q}^{M}\right\}\right]
\end{aligned}
$$

$$
\mathcal{A}_{B_{2}^{M}}^{f}=Y^{M} \sum_{i=q, \bar{q}} c_{i} \frac{\alpha_{s}}{2 \pi} \int_{x_{B}}^{\frac{x_{B}+\left(1-x_{B}\right) z}{1}} \frac{\mathrm{d} u}{u} \int_{z}^{1} \frac{\mathrm{d} \rho}{\rho} A(v(\rho, u))
$$$$
\cdot\left[f_{i / \mathrm{A}}^{r}\left(\frac{x_{B}}{u}, M_{f}^{2}\right) D_{\mathrm{h} / i}^{r}\left(\frac{z}{\rho}, M_{D}^{2}\right)\right.
$$$$
\cdot\left\{-\ln \frac{M_{f}^{2}}{Q^{2}} P_{q \leftarrow q}(u) \delta(1-\rho)-\ln \frac{M_{D}^{2}}{Q^{2}} P_{q \leftarrow q}(\rho) \delta(1-u)+C_{F} \Phi_{2 q q}^{M}\right\}
$$$$
+f_{i / \mathrm{A}}^{r}\left(\frac{x_{B}}{u}, M_{f}^{2}\right) D_{\mathrm{h} / g}^{r}\left(\frac{z}{\rho}, M_{D}^{2}\right)\left\{-\ln \frac{M_{D}^{2}}{Q^{2}} P_{g \leftarrow q}(\rho) \delta(1-u)+C_{F} \Phi_{2 q g}^{M}\right\}
$$$$
\left.+f_{g / \mathrm{A}}^{r}\left(\frac{x_{B}}{u}, M_{f}^{2}\right) D_{\mathrm{h} / i}^{r}\left(\frac{z}{\rho}, M_{D}^{2}\right)\left\{-\ln \frac{M_{f}^{2}}{Q^{2}} P_{q \leftarrow g}(u) \delta(1-\rho)+\frac{1}{2} \Phi_{2 g q}^{M}\right\}\right]
$$

$$
\begin{aligned}
\mathcal{A}_{C^{M}}^{f}= & Y^{M} \sum_{i=q, \bar{q}} c_{i} \frac{\alpha_{s}}{2 \pi} \int_{\frac{x_{B}}{1-\left(1-x_{B}\right) z}}^{1} \frac{\mathrm{d} u}{u} A(1) \\
& \cdot\left[M_{i, h / \mathrm{A}}^{r}\left(\frac{x_{B}}{u},\left(1-x_{B}\right) z, M_{f}^{2}\right)\left\{-\ln \frac{M_{f}^{2}}{Q^{2}} P_{q \leftarrow q}(u)\left(1-x_{B}\right)+C_{F} \Phi_{q}^{M}\right\}\right. \\
& \left.+M_{g, h / \mathrm{A}}^{r}\left(\frac{x_{B}}{u},\left(1-x_{B}\right) z, M_{f}^{2}\right)\left\{-\ln \frac{M_{f}^{2}}{Q^{2}} P_{q \leftarrow g}(u)\left(1-x_{B}\right)+\frac{1}{2} \Phi_{g}^{M}\right\}\right]
\end{aligned}
$$

$$
\begin{aligned}
\mathcal{A}_{B_{1}^{L}}^{f}= & Y^{L} \sum_{i=q, \bar{q}} c_{i} \frac{\alpha_{s}}{2 \pi} \int_{x_{B}}^{\frac{x_{B}}{x_{B}+\left(1-x_{B}\right) z}} \frac{\mathrm{d} u}{u} \int_{a(u)}^{1} \frac{\mathrm{d} \rho}{\rho} A(v(\rho, u)) \\
& \cdot\left[f_{i / \mathrm{A}}^{r}\left(\frac{x_{B}}{u}, M_{f}^{2}\right) D_{\mathrm{h} / i}^{r}\left(\frac{z}{\rho}, M_{D}^{2}\right) C_{F} \Phi_{1 q q}^{L}\right. \\
& +f_{i / \mathrm{A}}^{r}\left(\frac{x_{B}}{u}, M_{f}^{2}\right) D_{\mathrm{h} / g}^{r}\left(\frac{z}{\rho}, M_{D}^{2}\right) C_{F} \Phi_{1 q g}^{L} \\
& \left.+f_{g / \mathrm{A}}^{r}\left(\frac{x_{B}}{u}, M_{f}^{2}\right) D_{\mathrm{h} / i}^{r}\left(\frac{z}{\rho}, M_{D}^{2}\right) \frac{1}{2} \Phi_{1 g q}^{L}\right] \\
\mathcal{A}_{B_{2}^{L}[A}^{f}= & Y^{L} \sum_{i=q, \bar{q}} c_{i} \frac{\alpha_{s}}{2 \pi} \int_{\frac{x_{B}}{x_{B}+\left(1-x_{B}\right) z}}^{1} \frac{\mathrm{d} u}{u} \int_{z}^{1} \frac{\mathrm{d} \rho}{\rho} A(v(\rho, u))
\end{aligned}
$$




$$
\begin{aligned}
& \cdot\left[f_{i / \mathrm{A}}^{r}\left(\frac{x_{B}}{u}, M_{f}^{2}\right) D_{\mathrm{h} / i}^{r}\left(\frac{z}{\rho}, M_{D}^{2}\right) C_{F} \Phi_{2 q q}^{L}\right. \\
& +f_{i / \mathrm{A}}^{r}\left(\frac{x_{B}}{u}, M_{f}^{2}\right) D_{\mathrm{h} / g}^{r}\left(\frac{z}{\rho}, M_{D}^{2}\right) C_{F} \Phi_{2 q g}^{L} \\
& \left.+f_{g / \mathrm{A}}^{r}\left(\frac{x_{B}}{u}, M_{f}^{2}\right) D_{\mathrm{h} / i}^{r}\left(\frac{z}{\rho}, M_{D}^{2}\right) \frac{1}{2} \Phi_{2 g q}^{L}\right] \\
\mathcal{A}_{C^{L}}^{f}= & Y^{L} \sum_{i=q, \bar{q}} c_{i} \frac{\alpha_{s}}{2 \pi} \int_{\frac{x_{B}}{1-\left(1-x_{B}\right) z}}^{1} \frac{\mathrm{d} u}{u} A(1) \\
& \cdot\left[M_{i, h / \mathrm{A}}^{r}\left(\frac{x_{B}}{u},\left(1-x_{B}\right) z, M_{f}^{2}\right) C_{F} \Phi_{q}^{L}\right. \\
& \left.+M_{g, h / \mathrm{A}}^{r}\left(\frac{x_{B}}{u},\left(1-x_{B}\right) z, M_{f}^{2}\right) \frac{1}{2} \Phi_{g}^{L}\right]
\end{aligned}
$$

For some of the contributions the integration region is split into two parts, according to finite terms that arise from singular regions. The form given here should be suitable for numerical evaluation.

\section{Summary and Conclusions}

In this paper the one-particle inclusive cross section in deeply inelastic lepton-nucleon scattering has been expressed in terms of parton densities, fragmentation functions and fracture functions. A cut in the transverse momentum of the observed particle is not required. Compared to the case of a hadron that is well separated from the target remnant, additional collinear singularities are encountered because of the enlarged phase-space available to the parent parton of the observed particle. They stem from the phase-space region where the parent parton of the observed particle is collinear to the incoming parton. These singularities can, to $\mathcal{O}\left(\alpha_{s}\right)$, be consistently absorbed into the fracture functions. A check of the calculation is provided by the determination of the evolution equation of the fracture functions; it coincides with the one given in [14].

Fracture functions allow for a coherent and unified description of one-particle inclusive production processes in the case of incoming hadrons, without having to assume specific models for the target fragmentation [19]. As in the case of parton densities, the ignorance of the nucleon wave function is parametrized in a phenomenological distribution function, which has to measured. For target fragmentation, fracture functions have the advantage over phenomenological models that the correct scale dependence is built in, because of their evolution equation. It is expected that fracture functions are as universal as parton densities, i.e. that they can be determined in a specific process and then used for predictions in other processes.

The results of this paper should be consistent to $\mathcal{O}\left(\alpha_{s}\right)$ if the fracture functions are extracted at a scale $M_{0}^{2}$ and then used for predictions at a scale $M^{2}$, with $\ln \left(M^{2} / M_{0}^{2}\right)$ not too large. However, in order to evolve the fracture functions to scales $M^{2}$ very different from $M_{0}^{2}$, the evolution kernel (the analogon of the anomalous dimensions in the case of parton densities and fragmentation functions) should be known on the two-loop level. This effort would be necessary to have genuine $\mathcal{O}\left(\alpha_{s}\right)$ predictions in this case.

An interesting question would be to find out whether factorization of QCD subprocesses is always possible that collinear singularities can consistently be absorbed order by order into renormalized distribution functions, in the case that there is no transverse momentum cut for the observed particle. 
This would certainly be necessary to make the cross section well-defined. Another question is whether there is a connection with the formalism of Mueller [20, 21, 22] for one-particle inclusive processes. We hope to return to these questions in the future.

A more practical issue is the application of the concepts of [14] and the results of this paper to a real experimental situation, e.g. to deeply inelastic electron-proton scattering at HERA. An interesting application would be the "heavy-quark inclusive" cross section, i.e. the cross section for the production of a heavy quark plus anything. There is no obvious reason why the methods used in this paper should not be applicable to the production of heavy quarks instead of hadrons. In fact, explicit results for the fragmentation function of partons into heavy quarks are available [23], and similar results should be possible for fracture functions. Work along these lines is in progress.

\section{Acknowledgements}

I gratefully acknowledge disscussions with A. Ali, G. Altarelli, P. Nason, T. Sjöstrand and G. Veneziano. Moreover I wish to thank P. Nason and G. Veneziano for a critical reading of the manuscript. 


\section{A Phase-Space Parametrizations}

In this appendix three different parametrizations of the two-particle phase-space are given.

A) The integration variable $\rho$ is the energy $E_{1}$ of one of the partons in the $\left(\vec{P}_{0}+\vec{q}=\overrightarrow{0}\right)$ frame, scaled by the proton momentum,

$$
\rho=\frac{E_{1}}{E_{0}\left(1-x_{B}\right)}
$$

This parametrization is used for those contributions in which a collinear singularity has to be absorbed into a fragmentation function $D$; it is

$$
\begin{aligned}
\mathrm{dPS}^{(2)}= & \frac{1}{8 \pi} \frac{(4 \pi)^{\epsilon}}{\Gamma(1-\epsilon)}\left(Q^{2}\right)^{-\epsilon} \frac{u\left(1-x_{B}\right)}{u-x_{B}} \\
\cdot & \left(1-x_{B}\right)^{-2 \epsilon} u^{-\epsilon}(1-u)^{-\epsilon}\left(u-x_{B}\right)^{2 \epsilon}(\rho-a(u))^{-\epsilon}(1-\rho)^{-\epsilon} \mathrm{d} \rho .
\end{aligned}
$$

Here

$$
a(u)=\frac{x_{B}}{1-x_{B}} \frac{1-u}{u} .
$$

The energies and invariants are given by

$$
\begin{aligned}
E_{1} & =E_{0}\left(1-x_{B}\right) \rho \\
E_{2} & =E_{0}\left(1-x_{B}\right)(1-\rho+a(u)) \\
s_{12} & =Q^{2} \frac{1-u}{u} \\
s_{i 1} & =Q^{2} \frac{1-x_{B}}{u-x_{B}}(\rho-a(u)) \\
s_{i 2} & =Q^{2} \frac{1-x_{B}}{u-x_{B}}(1-\rho) .
\end{aligned}
$$

The angular variable $v_{1}=\left(1-\cos \vartheta_{1}\right) / 2$ is

$$
v_{1}=v(\rho, u)=\frac{x_{B}(1-u)}{u-x_{B}} \frac{1-\rho}{\rho} .
$$

The range of integration is restricted to $\rho \in[a(u), 1]$; however, in order to ensure that the energy of the parent parton of the observed hadron is larger than that of the hadron, the additional condition $\rho \geq z$ must be satisfied.

B) The integration variable $w$ is related to an angular variable in the CM system of the virtual photon and the incoming parton. Its relation to $\rho$ is

$$
w=\frac{1-\rho}{1-a(u)}
$$

This parametrization is used for the contributions that involve a fracture function; it is given by

$$
\operatorname{dPS}^{(2)}=\frac{1}{8 \pi} \frac{(4 \pi)^{\epsilon}}{\Gamma(1-\epsilon)}\left(Q^{2}\right)^{-\epsilon}(1-u)^{-\epsilon} u^{\epsilon}(w(1-w))^{-\epsilon} \mathrm{d} w
$$


The energies and invariants are

$$
\begin{aligned}
E_{1} & =E_{0}\left(1-x_{B}\right)(1-(1-a(u)) w) \\
E_{2} & =E_{0}\left(1-x_{B}\right)(a(u)+(1-a(u)) w) \\
s_{12} & =Q^{2} \frac{1-u}{u} \\
s_{i 1} & =Q^{2} \frac{1}{u}(1-w) \\
s_{i 2} & =Q^{2} \frac{1}{u} w .
\end{aligned}
$$

The angular variable $v_{1}$ is

$$
v_{1}=v(w, u)=\frac{a(u) w}{1-(1-a(u)) w} .
$$

The range of integration is restricted to $w \in[0,1]$.

C) This parametrization is convenient for the contributions in which the observed hadron originates from a parton that is collinear to the incoming parton. A variable $u^{\prime}$ is introduced by

$$
u^{\prime}=1-\frac{1-x_{B}}{x_{B}} \rho u
$$

The parametrization is given by

$$
\begin{aligned}
\mathrm{dPS}^{(2)}= & \frac{1}{8 \pi} \frac{(4 \pi)^{\epsilon}}{\Gamma(1-\epsilon)}\left(Q^{2}\right)^{-\epsilon} \frac{x_{B}}{u-x_{B}} \\
\cdot & \left(u-u^{\prime}\right)^{-\epsilon}(1-u)^{-\epsilon}\left(1-\frac{x_{B}}{1-x_{B}} \frac{1-u^{\prime}}{u}\right)^{-\epsilon}\left(u-x_{B}\right)^{2 \epsilon} x_{B}{ }^{-\epsilon}\left(1-x_{B}\right)^{-\epsilon} \mathrm{d} u^{\prime} .
\end{aligned}
$$

The energies and invariants are

$$
\begin{aligned}
& E_{1}=E_{0} x_{B} \frac{1-u^{\prime}}{u} \\
& E_{2}=E_{0}\left(1-x_{B}\right)\left(1-\frac{x_{B}}{1-x_{B}} \frac{u-u^{\prime}}{u}\right) \\
& s_{12}=Q^{2} \frac{1-u}{u} \\
& s_{i 1}=Q^{2} \frac{x_{B}}{u-x_{B}} \frac{u-u^{\prime}}{u} \\
& s_{i 2}=Q^{2} \frac{1-x_{B}}{u-x_{B}}\left(1-\frac{x_{B}}{1-x_{B}} \frac{1-u^{\prime}}{u}\right) .
\end{aligned}
$$

The angular variable $v_{1}$ is

$$
v_{1}=v\left(u^{\prime}, u\right)=\frac{1-u}{u-x_{B}} \frac{\left(1-x_{B}\right) u-x_{B}\left(1-u^{\prime}\right)}{1-u^{\prime}} .
$$

The range of integration is restricted to $u^{\prime} \in\left[1-\frac{1-x_{B}}{x_{B}} u, u\right]$. It has to be further restricted by

$$
u^{\prime} \leq 1-\frac{1-x_{B}}{x_{B}} z u
$$

in order to avoid that the outgoing hadron has a larger energy than its parent parton. 


\section{B Summary of Cross-Section Formulas}

In this appendix the expressions for the cross section including the divergent parts are written out. They are

$$
\begin{aligned}
& \mathcal{A}_{B_{1}^{M}}=Y^{M} \sum_{i=q, \bar{q}} c_{i} \frac{\alpha_{s}}{2 \pi} \\
& \cdot\left\{\int_{x_{B}}^{\frac{x_{B}}{x_{B}+\left(1-x_{B}\right) z}} \frac{\mathrm{d} u}{u} \int_{a(u)}^{1} \frac{\mathrm{d} \rho}{\rho} A(v(\rho, u))\right. \\
& \cdot\left[f_{i / \mathrm{A}}\left(\frac{x_{B}}{u}\right) D_{\mathrm{h} / i}\left(\frac{z}{\rho}\right)\right. \\
& \cdot\left\{-\frac{1}{\epsilon} \frac{\Gamma(1-\epsilon)}{\Gamma(1-2 \epsilon)}\left(\frac{4 \pi \mu^{2}}{M_{f}^{2}}\right)^{\epsilon} P_{q \leftarrow q}(u) \delta(1-\rho)\right. \\
& \left.-\ln \frac{M_{f}^{2}}{Q^{2}} P_{q \leftarrow q}(u) \delta(1-\rho)+C_{F} \Phi_{1 q q}^{M}\right\} \\
& +f_{i / \mathrm{A}}\left(\frac{x_{B}}{u}\right) D_{\mathrm{h} / g}\left(\frac{z}{\rho}\right) \\
& \left\{-\ln \frac{M_{f}^{2}}{Q^{2}} \hat{P}_{g q \leftarrow q}(u) \delta(\rho-a(u))+C_{F} \Phi_{1 q g}^{M}\right\} \\
& +f_{g / \mathrm{A}}\left(\frac{x_{B}}{u}\right) D_{\mathrm{h} / i}\left(\frac{z}{\rho}\right) \\
& \cdot\left\{-\frac{1}{\epsilon} \frac{\Gamma(1-\epsilon)}{\Gamma(1-2 \epsilon)}\left(\frac{4 \pi \mu^{2}}{M_{f}^{2}}\right)^{\epsilon} P_{q \leftarrow g}(u) \delta(1-\rho)\right. \\
& \left.\left.-\ln \frac{M_{f}^{2}}{Q^{2}} \hat{P}_{\bar{q} q \leftarrow g}(u) \delta(\rho-a(u))-\ln \frac{M_{f}^{2}}{Q^{2}} P_{q \leftarrow g}(u) \delta(1-\rho)+\frac{1}{2} \Phi_{1 g q}^{M}\right\}\right] \\
& +\int_{x_{B}}^{\frac{x_{B}}{x_{B}+\left(1-x_{B}\right) z}} \frac{\mathrm{d} u}{u}\left(1-x_{B}\right) A(1) \\
& \cdot\left[f_{i / \mathrm{A}}\left(\frac{x_{B}}{u}\right) D_{\mathrm{h} / g}\left(\frac{\left(1-x_{B}\right) z u}{x_{B}(1-u)}\right)\left(-\frac{1}{\epsilon}\right) \frac{\Gamma(1-\epsilon)}{\Gamma(1-2 \epsilon)}\left(\frac{4 \pi \mu^{2}}{M_{f}^{2}}\right)^{\epsilon} \frac{1}{1-u} \frac{u}{x_{B}} \hat{P}_{g q \leftarrow q}(u)\right. \\
& \left.\left.+f_{g / \mathrm{A}}\left(\frac{x_{B}}{u}\right) D_{\mathrm{h} / i}\left(\frac{\left(1-x_{B}\right) z u}{x_{B}(1-u)}\right)\left(-\frac{1}{\epsilon}\right) \frac{\Gamma(1-\epsilon)}{\Gamma(1-2 \epsilon)}\left(\frac{4 \pi \mu^{2}}{M_{f}^{2}}\right)^{\epsilon} \frac{1}{1-u} \frac{u}{x_{B}} \hat{P}_{\bar{q} q \leftarrow g}(u)\right]\right\} \\
& +\mathcal{O}(\epsilon) \\
& \mathcal{A}_{B_{2}^{M}}=Y^{M} \sum_{i=q, \bar{q}} c_{i} \frac{\alpha_{s}}{2 \pi} \int_{\frac{x_{B}}{x_{B}+\left(1-x_{B}\right) z}}^{1} \frac{\mathrm{d} u}{u} \int_{z}^{1} \frac{\mathrm{d} \rho}{\rho} A(v(\rho, u)) \\
& \cdot\left[f_{i / \mathrm{A}}\left(\frac{x_{B}}{u}\right) D_{\mathrm{h} / i}\left(\frac{z}{\rho}\right)\right. \\
& \left\{\frac{\Gamma(1-\epsilon)}{\Gamma(1-2 \epsilon)}\left(\frac{4 \pi \mu^{2}}{Q^{2}}\right)^{\epsilon} C_{F}\left(2 \frac{1}{\epsilon^{2}}+3 \frac{1}{\epsilon}\right) \delta(1-u) \delta(1-\rho)\right. \\
& -\frac{1}{\epsilon} \frac{\Gamma(1-\epsilon)}{\Gamma(1-2 \epsilon)}\left[\left(\frac{4 \pi \mu^{2}}{M_{f}^{2}}\right)^{\epsilon} P_{q \leftarrow q}(u) \delta(1-\rho)+\left(\frac{4 \pi \mu^{2}}{M_{D}^{2}}\right)^{\epsilon} P_{q \leftarrow q}(\rho) \delta(1-u)\right] \\
& \left.-\ln \frac{M_{f}^{2}}{Q^{2}} P_{q \leftarrow q}(u) \delta(1-\rho)-\ln \frac{M_{D}^{2}}{Q^{2}} P_{q \leftarrow q}(\rho) \delta(1-u)+C_{F} \Phi_{2 q q}^{M}\right\}
\end{aligned}
$$




$$
\begin{aligned}
& +f_{i / \mathrm{A}}\left(\frac{x_{B}}{u}\right) D_{\mathrm{h} / g}\left(\frac{z}{\rho}\right) \\
& \cdot\left\{-\frac{1}{\epsilon} \frac{\Gamma(1-\epsilon)}{\Gamma(1-2 \epsilon)}\left(\frac{4 \pi \mu^{2}}{M_{f}^{2}}\right)^{\epsilon} P_{g \leftarrow q}(\rho) \delta(1-u)\right. \\
& \left.\quad-\ln \frac{M_{D}^{2}}{Q^{2}} P_{g \leftarrow q}(\rho) \delta(1-u)+C_{F} \Phi_{2 q g}^{M}\right\} \\
& +f_{g / \mathrm{A}}\left(\frac{x_{B}}{u}\right) D_{\mathrm{h} / i}\left(\frac{z}{\rho}\right) \\
& \cdot\left\{-\frac{1}{\epsilon} \frac{\Gamma(1-\epsilon)}{\Gamma(1-2 \epsilon)}\left(\frac{4 \pi \mu^{2}}{M_{f}^{2}}\right)^{\epsilon} P_{q \leftarrow g}(u) \delta(1-\rho)\right. \\
& \left.\left.\quad-\ln \frac{M_{f}^{2}}{Q^{2}} P_{q \leftarrow g}(u) \delta(1-\rho)+\frac{1}{2} \Phi_{2 g q}^{M}\right\}\right] \\
& +\mathcal{O}(\epsilon) \quad-
\end{aligned}
$$

$$
\begin{aligned}
& \mathcal{A}_{C^{M}}=Y^{M} \sum_{i=q, \bar{q}} c_{i} \frac{\alpha_{s}}{2 \pi} \int_{\frac{x_{B}}{1-\left(1-x_{B}\right) z}}^{1} \frac{\mathrm{d} u}{u} A(1) \\
& \cdot\left[M_{i, h / \mathrm{A}}\left(\frac{x_{B}}{u},\left(1-x_{B}\right) z\right)\right. \\
& \cdot\left\{\frac{\Gamma(1-\epsilon)}{\Gamma(1-2 \epsilon)}\left(\frac{4 \pi \mu^{2}}{Q^{2}}\right)^{\epsilon} C_{F}\left(2 \frac{1}{\epsilon^{2}}+3 \frac{1}{\epsilon}\right) \delta(1-u)\left(1-x_{B}\right)\right. \\
& -\frac{1}{\epsilon} \frac{\Gamma(1-\epsilon)}{\Gamma(1-2 \epsilon)}\left(\frac{4 \pi \mu^{2}}{M_{f}^{2}}\right)^{\epsilon} P_{q \leftarrow q}(u)\left(1-x_{B}\right) \\
& \left.-\ln \frac{M_{f}^{2}}{Q^{2}} P_{q \leftarrow q}(u)\left(1-x_{B}\right)+C_{F} \Phi_{q}^{M}\right\} \\
& +M_{g, h / \mathrm{A}}\left(\frac{x_{B}}{u},\left(1-x_{B}\right) z\right) \\
& \left\{-\frac{1}{\epsilon} \frac{\Gamma(1-\epsilon)}{\Gamma(1-2 \epsilon)}\left(\frac{4 \pi \mu^{2}}{M_{f}^{2}}\right)^{\epsilon} P_{q \leftarrow g}(u)\left(1-x_{B}\right)\right. \\
& \left.\left.-\ln \frac{M_{f}^{2}}{Q^{2}} P_{q \leftarrow g}(u)\left(1-x_{B}\right)+\frac{1}{2} \Phi_{g}^{M}\right\}\right] \\
& +\mathcal{O}(\epsilon)
\end{aligned}
$$

$$
\begin{aligned}
\mathcal{A}_{B_{1}^{L}}= & Y^{L} \sum_{i=q, \bar{q}} c_{i} \frac{\alpha_{s}}{2 \pi} \int_{x_{B}}^{\frac{x_{B}}{x_{B}+\left(1-x_{B}\right) z}} \frac{\mathrm{d} u}{u} \int_{a(u)}^{1} \frac{\mathrm{d} \rho}{\rho} A(v(\rho, u)) \\
& \cdot\left[f_{i / \mathrm{A}}\left(\frac{x_{B}}{u}\right) D_{\mathrm{h} / i}\left(\frac{z}{\rho}\right) C_{F} \Phi_{1 q q}^{L}\right. \\
& +f_{i / \mathrm{A}}\left(\frac{x_{B}}{u}\right) D_{\mathrm{h} / g}\left(\frac{z}{\rho}\right) C_{F} \Phi_{1 q g}^{L} \\
& \left.+f_{g / \mathrm{A}}\left(\frac{x_{B}}{u}\right) D_{\mathrm{h} / i}\left(\frac{z}{\rho}\right) \frac{1}{2} \Phi_{1 g q}^{L}\right]+\mathcal{O}(\epsilon)
\end{aligned}
$$




$$
\begin{aligned}
\mathcal{A}_{B_{2}^{L}}= & Y^{L} \sum_{i=q, \bar{q}} c_{i} \frac{\alpha_{s}}{2 \pi} \int_{\frac{x_{B}}{x_{B}+\left(1-x_{B}\right) z}}^{1} \frac{\mathrm{d} u}{u} \int_{z}^{1} \frac{\mathrm{d} \rho}{\rho} A(v(\rho, u)) \\
& \cdot\left[f_{i / \mathrm{A}}\left(\frac{x_{B}}{u}\right) D_{\mathrm{h} / i}\left(\frac{z}{\rho}\right) C_{F} \Phi_{2 q q}^{L}\right. \\
& +f_{i / \mathrm{A}}\left(\frac{x_{B}}{u}\right) D_{\mathrm{h} / g}\left(\frac{z}{\rho}\right) C_{F} \Phi_{2 q g}^{L} \\
& \left.+f_{g / \mathrm{A}}\left(\frac{x_{B}}{u}\right) D_{\mathrm{h} / i}\left(\frac{z}{\rho}\right) \frac{1}{2} \Phi_{2 g q}^{L}\right]+\mathcal{O}(\epsilon) \\
\mathcal{A}_{C^{L}}= & Y^{L} \sum_{i=q, \bar{q}} c_{i} \frac{\alpha_{s}}{2 \pi} \int_{\frac{x_{B}}{1-\left(1-x_{B}\right) z}}^{1} \frac{\mathrm{d} u}{u} A(1) \\
& \cdot\left[M_{i, h / \mathrm{A}}\left(\frac{x_{B}}{u},\left(1-x_{B}\right) z\right) C_{F} \Phi_{q}^{L}\right. \\
& \left.+M_{g, h / \mathrm{A}}\left(\frac{x_{B}}{u},\left(1-x_{B}\right) z\right) \frac{1}{2} \Phi_{g}^{L}\right]+\mathcal{O}(\epsilon)
\end{aligned}
$$

Here the subtracted and unsubtracted Altarelli-Parisi splitting functions are [16, 24]:

$$
\begin{aligned}
P_{q \leftarrow q}(u) & =C_{F}\left[2\left(\frac{1}{1-u}\right)_{+}+\frac{3}{2} \delta(1-u)-1-u\right] \\
P_{q \leftarrow g}(u) & =\frac{1}{2}\left[1-2 u+2 u^{2}\right] \\
P_{g \leftarrow q}(u) & =C_{F}\left[2 \frac{1}{u}-2+u\right] \\
\hat{P}_{g q \leftarrow q}(u) & =C_{F}\left[2 \frac{1}{1-u}-1-u\right] \\
\hat{P}_{\bar{q} q \leftarrow g}(u) & =\frac{1}{2}\left[1-2 u+2 u^{2}\right]
\end{aligned}
$$




\section{Explicit Formulas for the Finite Contributions}

In this appendix the explicit expressions for the finite contributions are presented. They are

$$
\begin{aligned}
& \Phi_{1 q q}^{M}=\delta(1-u) \delta(1-\rho) \frac{\pi^{2}}{3} \\
& +\delta(1-\rho)\left[2\left(\frac{\ln (1-u)}{1-u}\right)_{+u[0, \underline{1}]}+1-u-(1+u) \ln (1-u)-\frac{1+u^{2}}{1-u} \ln \frac{u-x_{B}}{1-x_{B}}\right] \\
& +\delta(1-u)\left[2\left(\frac{\ln (1-\rho)}{1-\rho}\right)_{+\rho[0, \underline{1}]}+1-\rho-(1+\rho) \ln (1-\rho)+\frac{1+\rho^{2}}{1-\rho} \ln \rho\right] \\
& +2\left(\frac{1}{1-\rho}\right)_{+\rho[0, \underline{1}]}\left(\frac{1}{1-u}\right)_{+u[0, \underline{1}]}-\left(\frac{1}{1-u}\right)_{+u[0, \underline{1}]}(1+\rho)-\left(\frac{1}{1-\rho}\right)_{+\rho[0, \underline{1}]}(1+u) \\
& +(1-\rho) \frac{x_{B}}{u-x_{B}}\left(1+\frac{u\left(1-x_{B}\right)}{u-x_{B}}\right)-2 \frac{u x_{B}}{u-x_{B}}+2 \\
& \Phi_{1 q g}^{M}=\delta(\rho-a(u))\left[1-u+\frac{1+u^{2}}{1-u} \ln \frac{1-u}{u}\right] \\
& +\left(\frac{1}{\rho-a(u)}\right)_{+\rho[\underline{a(u), 1]}} \frac{1+u^{2}}{1-u}+\frac{\left(1-x_{B}\right)^{2}}{\left(u-x_{B}\right)^{2}} \frac{u^{2}}{1-u} \rho-\frac{x_{B}\left(1-x_{B}\right)}{\left(u-x_{B}\right)^{2}} u-2 \frac{1-x_{B}}{u-x_{B}} \frac{u^{2}}{1-u} \\
& \Phi_{1 g q}^{M}=\delta(\rho-a(u))\left[2 u(1-u)+\left(1-2 u+2 u^{2}\right) \ln \frac{1-u}{u}\right] \\
& +\delta(1-\rho)\left[2 u(1-u)+\left(1-2 u+2 u^{2}\right) \ln \frac{(1-u)\left(1-x_{B}\right)}{u-x_{B}}\right] \\
& +\left(\frac{1}{\rho-a(u)}\right)_{+\rho[\underline{a(u)}, 1]}\left(1-2 u+2 u^{2}\right)+\left(\frac{1}{1-\rho}\right)_{+\rho[0, \underline{1}]}\left(1-2 u+2 u^{2}\right)-2 \frac{1-x_{B}}{u-x_{B}} u \\
& \Phi_{2 q q}^{M}=\Phi_{1 q q}^{M} \\
& \Phi_{2 q g}^{M}=\delta(1-u)\left[\rho+(\ln \rho+\ln (1-\rho))\left(\rho+\frac{2}{\rho}-2\right)\right] \\
& +\left(\frac{1}{1-u}\right)_{+u[0, \underline{1}]}\left(\rho+\frac{2}{\rho}-2\right) \\
& +2-2 \frac{x_{B} u}{u-x_{B}}+\frac{x_{B}}{u-x_{B}} \rho-\frac{x_{B}\left(1-x_{B}\right) u}{\left(u-x_{B}\right)^{2}}(1-\rho)+\frac{1}{\rho-a(u)} \frac{1+u^{2}}{1-u}-2 \frac{1}{\rho} \frac{1}{1-u} \\
& \Phi_{2 g q}^{M}=\delta(1-\rho)\left[2 u(1-u)+\left(1-2 u+2 u^{2}\right) \ln \frac{(1-u)\left(1-x_{B}\right)}{u-x_{B}}\right] \\
& +\frac{1}{\rho-a(u)}\left(1-2 u+2 u^{2}\right)+\left(\frac{1}{1-\rho}\right)_{+\rho[0, \underline{1}}\left(1-2 u+2 u^{2}\right)-2 \frac{1-x_{B}}{u-x_{B}} u \\
& \Phi_{q}^{M}=\left(1-x_{B}\right)\left[\frac{7}{2} \delta(1-u)-\frac{3}{2}\left(\frac{1}{1-u}\right)_{+u[0, \underline{1}}+2\left(\frac{\ln (1-u)}{1-u}\right)_{+u[0, \underline{1}]}\right. \\
& \left.+3-u-(1+u) \ln (1-u)-\frac{1+u^{2}}{1-u} \ln u\right]
\end{aligned}
$$




$$
\begin{aligned}
& \Phi_{g}^{M}=\left(1-x_{B}\right)\left(\ln \frac{1-u}{u}-1\right)\left(1-2 u+2 u^{2}\right) \\
& \Phi_{1 q q}^{L}=2 \frac{\left(1-x_{B}\right)^{2}}{\left(u-x_{B}\right)^{2}} u^{3} \rho-2 \frac{x_{B}\left(1-x_{B}\right)}{\left(u-x_{B}\right)^{2}} u^{2}(1-u) \\
& \Phi_{1 q g}^{L}=2 \frac{\left(1-x_{B}\right)^{2}}{\left(u-x_{B}\right)^{2}} u^{3}(1-\rho) \\
& \Phi_{1 g q}^{L}=4 \frac{1-x_{B}}{u-x_{B}} u^{2}(1-u) \\
& \Phi_{2 q q}^{L}=\Phi_{1 q q}^{L} \\
& \Phi_{2 q g}^{L}=\Phi_{1 q g}^{L} \\
& \Phi_{2 g q}^{L}=\Phi_{1 g q}^{L} \\
& \Phi_{q}^{L}=\left(1-x_{B}\right) 2 u(1-u) \\
& \Phi_{B}^{L}=\left(1-x_{B}\right) u
\end{aligned}
$$




\section{References}

[1] H.D. Politzer, Phys. Lett. $\underline{70 B}$ (1977) 430

[2] D. Amati, R. Petronzio, G. Veneziano, Nucl. Phys. B140 (1978) 54 and B146 (1978) 29

[3] R.K. Ellis, H. Georgi, M. Machcek, H.D. Politzer, G.G. Ross, Phys. Lett. $\underline{78 B}$ (1978) 281

[4] R.K. Ellis, H. Georgi, M. Machcek, H.D. Politzer, G.G. Ross, Nucl. Phys. B152 (1979) 285

[5] G. Altarelli, R.K. Ellis, G. Martinelli, S.Y. Pi, Nucl. Phys. B160 (1979) 301

[6] R. Baier, K. Fey, Z. Phys. C2 (1979) 339

[7] W. Furmanski, R. Petronzio, Z. Phys. C11 (1982) 293

[8] A. Mendez, Nucl. Phys. B145 (1978) 199

[9] A. Mendez, A. Raychaudhuri, V.J. Stenger, Nucl. Phys. B148 (1979) 499

[10] N. Sakai, Phys. Lett. $\underline{85 \mathrm{~B}}(1979) 67$

[11] P. Chiappetta, Y. Gabellini, T. Grandon, J.L. Meunier, Nucl. Phys. B239 (1984) 410

[12] P. Chiappetta, J.P. Guillet, Z. Phys. C32 (1986) 209

[13] E.L. Berger, in: Proceedings of the NPAS Workshop 1987, Stanford

[14] L. Trentadue, G. Veneziano, Phys. Lett. B323 (1994) 201

[15] R.P. Feynman, Photon Hadron Interactions, W.A. Benjamin, Inc. (Reading, Mass., 1972)

[16] G. Altarelli, R.K. Ellis, G. Martinelli, Nucl. Phys. B157 (1979) 461

[17] Ch. Rumpf, G. Kramer, J. Willrodt, Z. Phys. C67 (1981) 337

[18] I.M. Gel'fand, G.E. Shilov, Generalized Functions, Vol. I, Academic Press (New York, London, 1964)

[19] A. Bartl, H. Fraas, W. Majerotto, Phys. Rev. D26 (1982) 1061

[20] A.H. Mueller, Phys. Rev. D2 (1970) 2963

[21] R.N. Cahn, E.W. Colglazier, Phys. Rev. D8 (1973) 3019

[22] D. Horn, F. Zachariasen, Hadron Physics at Very High Energies, W. Benjamin, Inc. (Reading, Mass., 1973), Chapter 6

[23] B. Mele, P. Nason, Nucl. Phys. B361 (1991) 626

[24] K. Konishi, A. Ukawa, G. Veneziano, Nucl. Phys. B157 (1979) 45 


\section{Figure Captions:}

Fig. 1 Contributions to the one-particle inclusive cross section: current (a), target (b). $f$ stands for the parton densities, $M$ for fracture functions and $P$ for any hard process.

Fig. 2 The parton-model process for the current contribution (a) and target contribution (b). $D$ is the fragmentation function.

Fig. 3 The virtual corrections to $\mathcal{O}\left(\alpha_{s}\right)$.

Fig. 4 The real corrections to $\mathcal{O}\left(\alpha_{s}\right)$ : diagrams contributing to the terms $B_{\alpha}^{A}$ (a) and $C^{A}$ (b). 
This figure "fig1-1.png" is available in "png" format from: http://arxiv.org/ps/hep-ph/9406274v1 
This figure "fig1-2.png" is available in "png" format from: http://arxiv.org/ps/hep-ph/9406274v1 
This figure "fig1-3.png" is available in "png" format from: http://arxiv.org/ps/hep-ph/9406274v1 

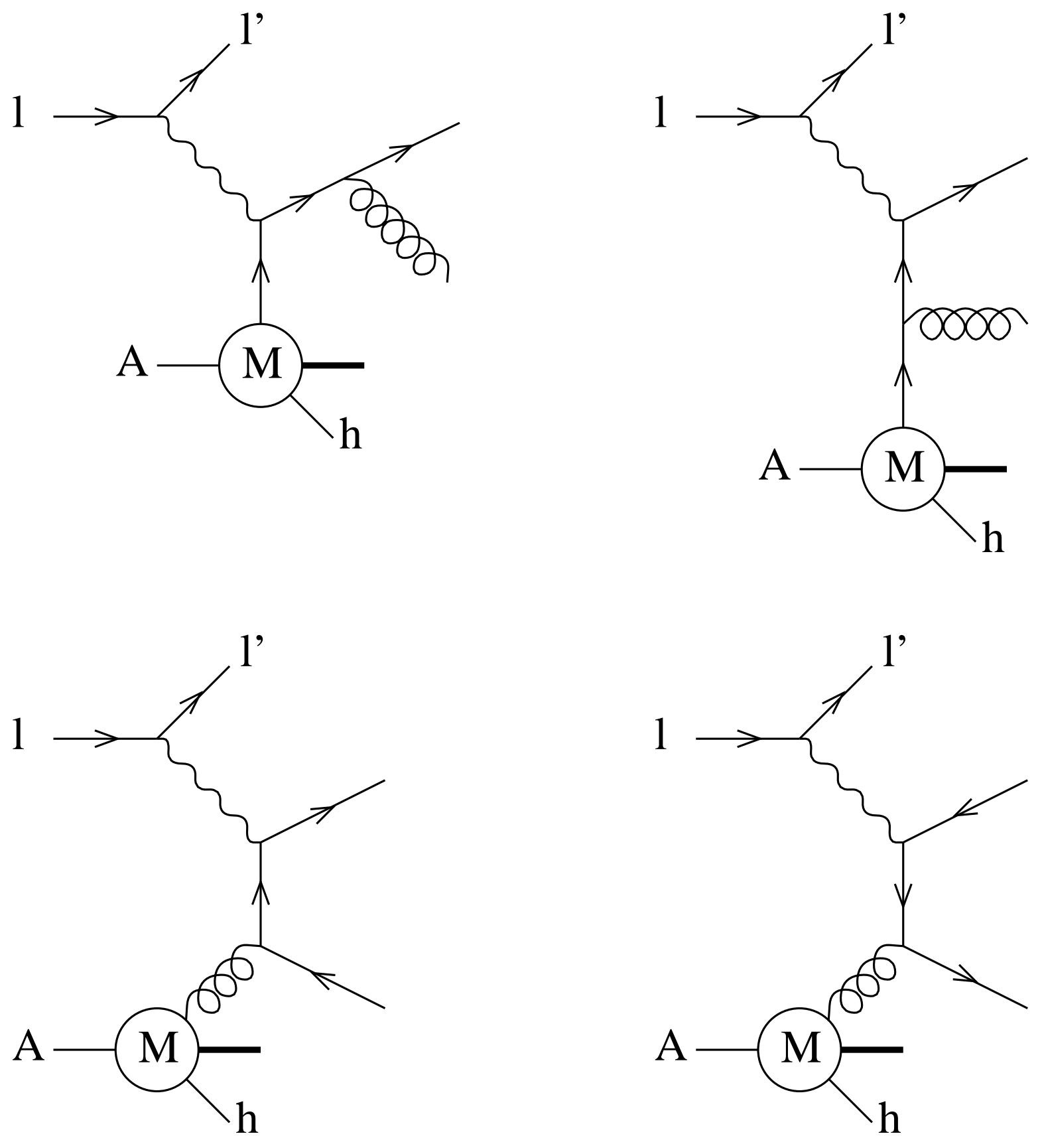

Fig. 4 (b) 